\title{
CONF-970592--2 \\ Design Optimization of a Liquid-Distribution Chamber-Slot Die Using the DAKOTA Toolkit*
}

\author{
Ken S. Chen and Walter R. Witkowski \\ Sandia National Laboratories, P.O. Box 5800, Albuquerque, NM 87185-6826 1997 \\ OSTI
}

\begin{abstract}
In the present study of design optimization of a liquid-distribution chamber-slot die, the DAKOTA (Design Analysis Kit for OpTimizAtion) toolkit, which is being developed by Sandia National Laboratories, was employed to navigate the search for the optimal die shape. This shape minimizes nonuniformity of flow at the slot exit for a given set of liquid properties and operating conditions. Three-dimensional, steady Newtonian-liquid flow fields inside the chamber-slot die were computed using FIDAP, a commercial computer code based on the finite element method. The objective function of flow nonuniformity at the slot exit is formulated as the percentage of coating material across the slot width having local-flowrate deviation greater than $1 \%$ from the mean. Computation of the objective function requires the integration of the velocity profile over the outflow plane. Two constraints, namely maximum hydrodynamic pressure and average residence time, were imposed in the optimization problem. The modified method of feasible directions algorithm was used to optimize the die geometry and to reduce the flow nonuniformity at the slot exit from $16.5 \%$ (initial design) to $3.2 \%$ (final design) for the chosen liquid properties and process conditions. Our case study demonstrates that liquid-distribution chamber-slot dies can be systematically optimized using DAKOTA.
\end{abstract}

\section{Introduction}

In premetered coating flows such as slide, slot, and curtain coating flows, which are the chief methods in manufacturing thin-film-coating products like video tapes and color photographic films, chamber-slot dies are usually employed to distribute coating liquids uniformly in the transverse or crossweb direction ${ }^{1,2}$. Similar liquid-distribution dies are also widely used in polymeric thin-film extrusion operations. Qualities of acceptable film or coating products often require that the cross-web nonuniformity be below a few percent.
Therefore, die geometry must be carefully designed and die machining must be painstakingly carried out to ensure that the desired flow uniformity at the slot exit be achieved. Die manufacturing is a time-consuming and costly operation because of the high precision requirement. Typically, coating solution is pumped into a chamber through a feed pipe, and is then extruded out through a slot (e.g., five feet wide and 0.25 millimeter high). Flow in the chamber-slot die must be laminar and the flowing coating liquid ranges from mildly shear-thinning to viscoelastic. The outflow uniformity is highly sensitive to a number of design parameters, including chamber shape, width, height and length, slot length and height, volumetric flowrate, liquid viscosity and density. Other factors affecting the out-flow uniformity include chamber and slot surface roughness and dimensional stability (slot height can be widened by hydrodynamic pressure when sufficiently high due to deflection). Constraints on the design problem may include ease of machining, weight, cost, maximum hydrodynamic pressure and residence time of fluid elements inside the die. The objective of our present study is to efficiently determine the optimal combination of die geometry, subject to the constraints of maximum pressure and average residence time, which yields the most uniform out-flow uniformity without having to carry out costly and time-consuming flow experiments. In scope, the present study is limited to isothermal steady Newtonian liquid chamber flow in an infinite-cavity die, in which chamber and slot shapes are uniform across the die width and the chamber cross-section is large enough such that the slot gap has dominant influence on slot-exit flow uniformity.

\section{Objective Function and Constraints}

Figure 1 schematically shows the flow-domain geometry of a chamber-slot liquid-distribution die. Here, six parameters are used to describe the chamber and slot geometries: slot height $\left(H_{s}\right)$, slot length $\left(L_{s}\right)$, chamber length $\left(L_{c}\right)$, chamber height $\left(H_{c}\right)$, slot-entrance inclination angle $(\alpha)$, and elevation of the

* DAKOTA (Design Analysis Kit for OpTimizAtion) tookit is being developed by Sandia National Laboratories for performing optimization of complex engineering processes, simulations of which involve large scale and CPU-intensive computations.

This work was supported by the United States Department of Energy under contract DE-AC04-94AL85000.

Sandia is a multiprogram laboratory operated by Sandia Corporation, a Lockheed Martin Company, for the United States Department of Energy.

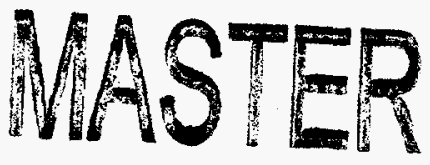




\section{DISCLAIMER}

This report was prepared as an account of work sponsored by an agency of the United States Government. Neither the United States Government nor any agency thereof, nor any of their employees, make any warranty, express or implied, or assumes any legal liability or responsibility for the accuracy, completeness, or usefulness of any information, apparatus, product, or process disclosed, or represents that its use would not infringe privately owned rights. Reference herein to any specific commercial product, process, or service by trade name, trademark, manufacturer, or otherwise does not necessarily constitute or imply its endorsement, recommendation, or favoring by the United States Government or any agency thereof. The views and opinions of authors expressed herein do not necessarily state or reflect those of the United States Government or any agency thereof. 


\section{DISCLAMMER}

Portions of this document may be illegible in electronic image products. Images are produced from the best available original document. 
feed-pipe relative to the die-bottom surface $\left(\mathrm{H}_{\mathrm{f}}\right)$. The die width (W) is fixed and is not considered as a design parameter in our optimization problem formulation. For simplicity, we consider a Newtonian coating liquid and a steady isothermal flow. Accordingly, flows in the chamber and slot are governed by the familiar Navier-Stokes equations. Relevant boundary conditions are: 1) on solid walls, no slip condition is applied, i.e., all three velocity components are zero. 2) on the symmetry plane (to reduce computational costs, we consider only half of the chamber-slot flow domain), the normal velocity component vanishes. 3 ) on the inflow plane, a fully developed duct Poiseuille flow is specified. 4) on the outflow plane, the flow is taken to be free of traction and the pressure is that of ambient. Further details of the three-dimensional chamber-slot flow model used in the present analysis are available elsewhere ${ }^{2}$. The three-dimensional, steady Newtonian-liquid flow fields inside the chamber-slot die were computed using FIDAP, a commercial computer code based on the finite element method ${ }^{3}$.

The desired objective function describes the percentage of slot or die width having a local flowrate deviation from the maximum within one percent at the outflow plane. To construct such an objective function, we first calculate the flowrate per unit slot width, $\mathrm{q}$, as follows:

$$
q(z)=\int_{\frac{H_{s}}{2}}^{\frac{H_{s}}{2}} u\left(x_{\text {outflow }}, y, z\right) d y
$$

where $\mathrm{u}$ is velocity; $\mathrm{H}_{\mathrm{s}}$ is slot height; $\mathrm{z}$ is the coordinate axis along the slot width, $\mathrm{y}$ along slot height, and $\mathrm{x}$ along slot length. Defining $d(z)$ as the percent deviation of local flowrate from its maximum,

$$
d(z)=\frac{q_{\max }-q(z)}{q_{\max }} 100 \%
$$

and denoting $z_{1}$ as the location at which $d\left(z=z_{1}\right)=1 \%$, our objective function can be calculated as follows:

$$
f_{\text {objective }}=\frac{\left|z_{1}\right|}{w / 2} 100 \%
$$

where $w$ is the slot or die width (Note: the symmetry plane is located at $\mathrm{z}=0$ ). For an optimal design, $\mathrm{f}_{\text {objective }}$ should be maximized. In other words, $f_{\text {objective }}$ should be as close to $100 \%$ as possible but never achieved due to edge effects.

One constraint in our die-design optimization problem is the maximum allowable hydrodynamic pressure, which controls the allowable deffection of the metal chamber and slot walls. Hydrodynamic pressure is chosen as the constraint here because slight slot height variation can cause large flow nonuniformity at the slot exit. The maximum value is used because it can be easily determined from the numericallycomputed discrete pressure field.

Another constraint is the average residence time of liquid elements at the slot exit. This constraint limits the average age of liquid elements in the chamber-slot die. Once the flow field (velocity and pressure) of the chamber-slot flow domain is obtained, residence times of liquid elements at each nodal points of the outflow plane can be calculated easily (using FIDAP's FIPOST module). the average residence time of liquid elements as they exit the slot can then be determined as follows:

$$
t_{\text {avg }}=\frac{\int_{-W / 2}^{W / 2} \int_{-H_{s} / 2}^{H_{s} / 2} t(y, z) d y d z}{W H_{s}}
$$

where $t(y, z)$ is the discrete residence-time function evaluated at the outflow plane and $t_{\text {avg }}$ is the average residence time of all liquid elements exiting the slot.

\section{Optimization Algorithm and Implementation}

The DAKOTA toolkit contains a set of iteration methods that can be used for optimization, non-deterministic and parameter studies. For the presented study, DAKOTA was used to interface the flow-field solver, FIDAP with DOT, which is a software package that contains a suite of various numerical optimization routines ${ }^{4}$. DOT's modified method of feasible directions routine was used to minimize the nonuniformity objective function described earlier. In the actual calculations, the objective function of nonuniformity, i.e., $\left(100-f_{o b}\right.$ jective), was minimized, which is equivalent to $f_{\text {objective }}$ being maximized $\left(f_{\text {objective }}\right.$ is the uniformity objective function as defined by Equation 3). DAKOTA uses an object-oriented design to interface analysis codes such as FIDAP and optimization routines such as DOT. The information transfer between DAKOTA/DOT and FIDAP occurs through I/O filter modules.

The input filter takes the design variable parameters specified by the optimization routine and generates the appropriate input data files to be used in the FIDAP flow-field solver. This requires preprocessing of a parameterized geometry template file with the specified design variables. Following the FIDAP analysis (i.e., after the flow field is computed), an output filter is then run or executed, which accomplishes the following tasks: i) read the velocity and pressure profiles from the FIDAP result-data files; ii) integrates the velocity data to calculate the aforementioned objective function; iii) computes the constraint values based on maximum pressure and average residence time; and iv) return these values to DAKOTA. The optimization routine then determines a new set of design variables for the analysis code and this process is continued until an optimal solution is obtained (i.e., a pre- 
scribed convergence criterion is met).

\section{Results and Discussion}

Figure 2 shows the initial die geometry from which our optimization iteration was started and the finite element mesh used in our flow-field calculations. This mesh has 980 brick elements, which was refined from a coarser model with 540 elements. Though it was much more CPU time intensive, we found it necessary to employ the refined mesh of 980 brick elements to eliminate kinks or nonsmoothness in the objective function. With the refined mesh, it took about $50 \mathrm{~min}-$ utes of CPU time for each objective function evaluation on a IBM SP 2 computer. A total of 21 runs (or objective-function evaluations) were necessary to arrive at the optimized design or die geometry shown in Figure 3.

In this case study, we considered low-viscosity coating liquids that are typically used in precision premetered coating processes. Specifically, the coating solution viscosity $(\mu)$ and density ( $p$ ) were chosen to be $15 \mathrm{mPa}$ (i.e., $15 \mathrm{cP}$ ) and 1000 $\mathrm{kg} / \mathrm{m}^{3}$, respectively. The average volumetric flowrate per unit slot width $\left(\mathrm{q}_{\mathrm{avg}}\right.$ ) was $1.5 \times 10^{-4} \mathrm{~m}^{2} / \mathrm{s}$. Moreover, the characteristic length scale used in calculating dimensionless quantities in the flow-field computations was chosen to be $10^{-3} \mathrm{~m}$. Using these conditions, the resultant Reynolds number $(\operatorname{Re}=$ $\left.\rho q_{a v g} / \mu\right)$ is 10 , which is well within the laminar flow regime that is necessary to produce a uniform flow across the die width at the slot exit. The constraint of maximum pressure was set to a dimensionless value of 2100 , corresponding to a dimensional value of $0.7 \mathrm{psi}$. Similarly, the constraint of maximum residence time was set to a dimensionless value of 325 , corresponding to a dimensional value of 2.2 seconds.

These values were chosen somewhat arbitrarily based on the nominal design to prevent overly large design changes.

DOT's modified method of feasible directions optimizer was used to successfully optimize the die geometry (with the initial guess shown in Figure 2) and reduce the flow nonuniformity at the slot exit from $16.5 \%$ to $3.2 \%$ (i.e. the percentage of die width having a local flowrate deviation from the maximum greater than one percent was reduced from 16.5 to 3.2 ). The final die geometry is displayed in Figure 3. Initial and final die dimensions and constraint values are listed in Table 1 (dimensions that were driven to their upper or lower bounds are indicated with *). A comparison between the initial and final geometries shows that the optimized die geometry is about twice as large as the initial geometry. As expected, slot height was reduced to its lower bound, thereby causing the maximum pressure to increase to a dimensionless value of 1832 (corresponding to a dimensional value of $0.6 \mathrm{psi}$ ). Because neither the pressure constraint nor the residence time constraint was active, the optimal design computed is not a direct function of the allowables chosen for these parameters. This low-pressure optimal solution is a result of the low viscosity value, which is typical in precision premetered coating processes. The maximum pressure is expected to be much greater in coating/extrusion processes involving high-viscosity liquids (e.g., adhesive coating, hotmelt extrusion).

\section{Concluding Remarks}

Numerical optimization of a liquid-distribution chamber-slot die using the modified method of feasible direction algorithm available in the DAKOTA optimization toolkit was demonstrated. It is evident that the die-design optimization effort has just begun. Continuation of this work includes investigation of high-viscosity coating liquids, which are expected to render the maximum pressure and average residence time constraints active. Also, instead of parameterizing with only a slot entrance angle, a more complex parameterization of the die chamber will be used to describe a teardrop chamber shape. Lastly, efforts are under way to incorporate analytical sensitivity capability within the FIDAP analysis code so that optimization efficiency can be evaluated comparatively for analytical-gradient-based, finitedifference-gradient-based, and pattern search approaches.

\section{References}

1. Sartor, L. 1990 Ph. D. Thesis, Univ. of Minnesota, Available from University Microfilms International, Ann Arbor, MI 48106.

2. Chen, K. S. 1992 Ph. D. Thesis, University of Minnesota. Available from UMI, Ann Arbor, MI48106.

3. Fluid Dynamics International 1993 FIDAP Users Manual, Version 7.0, Fluid Dynamics International, Evanston, IL.

4.Vanderplaats, Miura and Associates 1993 DOT Users Manual, Version 4.00, VMA Engineering, Goleta, CA

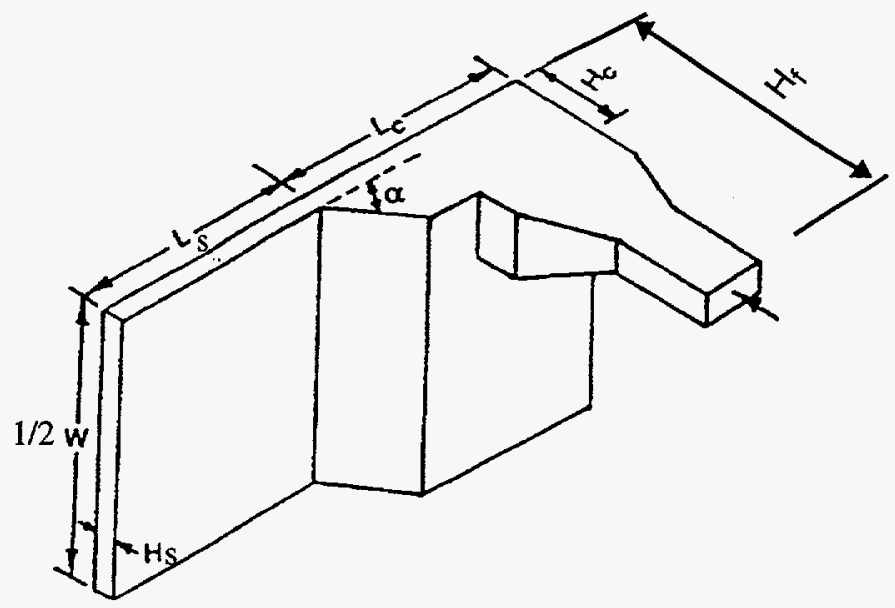

Figure 1. Geometry parameterization and design variables of a chamber-slot die (die width $W$ is fixed). 
Table 1: Preliminary optimization results for a case study

\begin{tabular}{|r|r|r|}
\hline & Initial & Final \\
\hline \hline Slot length, Ls (cm) & 5.0 & 11.3 \\
\hline Slot height, Hs (cm) & 1.0 & $0.25^{*}$ \\
\hline Slot Entrance Angle, $\alpha$ (degree) & 53.0 & $60.0^{*}$ \\
\hline Chamber length, Lc (cm) & 10.9 & $20.0^{*}$ \\
\hline Chamber height, Hc (cm) & 4.3 & 9.9 \\
\hline Feed-channel height, Hf (cm) & 15.0 & 14.3 \\
\hline $\begin{array}{r}\text { Average residence time at die } \\
\text { exit (dimensionless values) }\end{array}$ & 253.6 & 215.2 \\
\hline $\begin{array}{r}\text { Maximum pressure within die } \\
\text { (dimensionless values) }\end{array}$ & 42.3 & 1832.0 \\
\hline
\end{tabular}

* active bound constraint

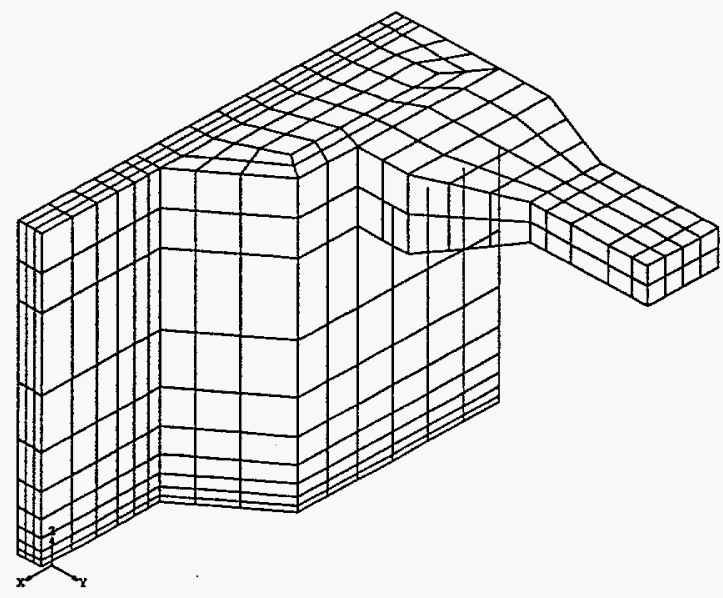

Figure 2. Initial die geometry in form of a finite element mesh. 


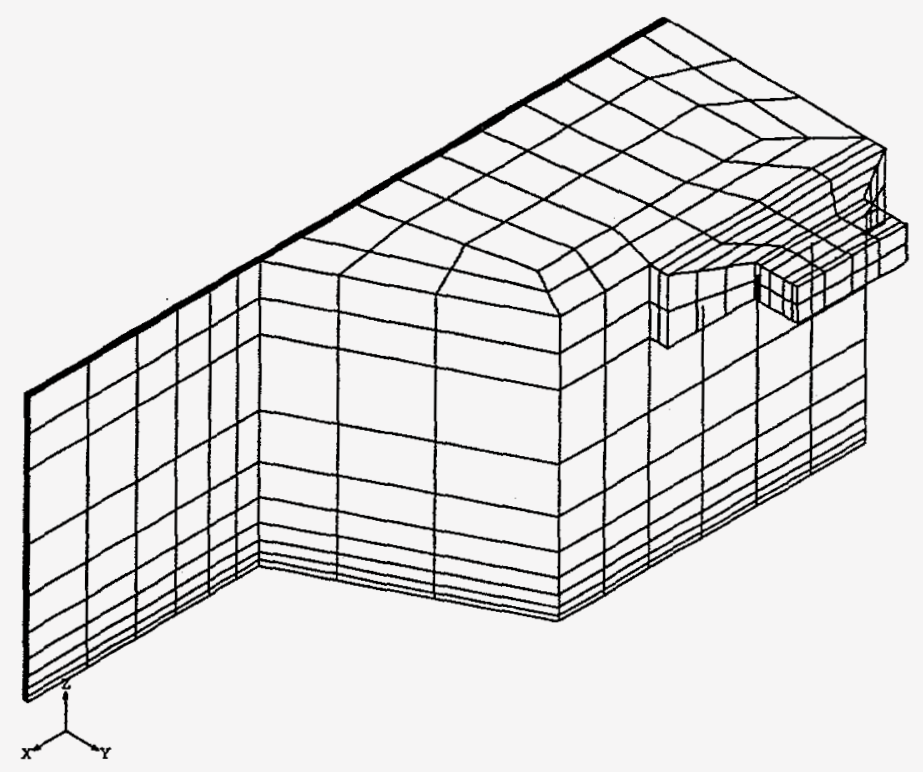

Figure 3. Final or optimal die geometry in form of a finite element mesh. 